\title{
Narrative review of developing new biomarkers for decision making in advanced testis cancer
}

\author{
Lucia Nappi $^{1,2} \wedge$, Craig Nichols ${ }^{3,4}$, Christian Kollmannsberger ${ }^{1}$ \\ ${ }^{1}$ Division of Medical Oncology, British Columbia Cancer - Vancouver Cancer Centre, Vancouver, BC, Canada; ${ }^{2}$ Department of Urologic Sciences, \\ Vancouver Prostate Centre, University of British Columbia, Vancouver, BC, Canada; ${ }^{3}$ Testicular Cancer Commons, Beaverton, Oregon, USA; \\ ${ }^{4}$ SWOG Group Chair's Office, Portland, OR, USA \\ Contributions: (I) Conception and design: All authors; (II) Administrative support: All authors; (III) Provision of study materials or patients: None; \\ (IV) Collection and assembly of data: All authors; (V) Data analysis and interpretation: All authors; (VI) Manuscript writing: All authors; (VII) Final \\ approval of manuscript: All authors. \\ Correspondence to: Lucia Nappi, MD, PhD, FRCPC. Division of Medical Oncology, British Columbia Cancer - Vancouver Cancer Centre, Vancouver, \\ BC, Canada; Department of Urologic Sciences, Vancouver Prostate Centre, University of British Columbia, 600 West 10th Ave Vancouver BC \\ V5Z4E6, Canada. Email: lucia.nappi@bccancer.bc.ca.
}

\begin{abstract}
Management of testicular germ cell tumor (GCT) patients is based on clinical determinants, mainly CT scan and serum tumor markers (alpha-fetoprotein, beta subunit of HCG and LDH). Treatment decisions are usually straightforward for patients with clear evidence of metastatic disease, confirmed either by imaging tests or by unequivocal elevated tumor markers. However, there are several clinical scenarios where the assessment of metastatic disease is complicated by the limited specificity of the current imaging tests and serum tumor markers. These include patients with clinical stage IIA GCT with negative tumor markers and patients with post-chemotherapy residual disease where, in absence of clear indicators of GCT, decision making and patient treatment allocation become challenging. Therefore, more accurate biomarkers are critical to reduce the risk of under-or over-treatment and to always deliver the most optimal therapy. The objectives of this narrative review are to review the available publications about micro-RNAs in GCT s and their potential clinical applications. Two clusters of micro-RNAs, miR-371a-3p and miR-302/367, specifically expressed by both seminoma and non-seminoma GCT and easily detectable in the peripheral blood, have demonstrated to be promising in this endeavor. Large prospective trials are ongoing to define the operating characteristics of these biomarkers and their clinical utility to improve GCT patient management and reduce the error rate deriving from clinical uncertainty, therefore reducing the risk of sub-optimal treatments.
\end{abstract}

Keywords: Biomarkers; micro-RNAs; miR-371a-3p; testicular germ cell tumors (testicular GCTs)

Submitted Sep 09, 2020. Accepted for publication Nov 30, 2020.

doi: $10.21037 / \mathrm{tau}-20-1246$

View this article at: http://dx.doi.org/10.21037/tau-20-1246

\section{Introduction}

Testicular germ cell tumors (GCT) are highly curable malignancies and represent one of the few successful stories in the history of medical oncology $(1,2)$. The characteristics of the patients population and the high cure rate achieved with chemotherapy treatment make this tumor unique (3).
First, the goal of care is always cure and the chances for cure are highest if the appropriate treatments are delivered correctly and in a very standardized way $(4,5)$. Second, cure should be achieved with as much reduction of short and particularly long-term toxicity as possible $(6,7)$. This is important due to the young age of the GCT patients

^ ORCID: 0000-0002-4365-7251. 
and the long-life expectancy following curative therapy. Chemotherapy and radiation therapy are associated with acute and long-term toxicity that affect the lifespan but also the quality of life of the GCT survivors (8-12).

Although adjuvant chemotherapy is an option in patients with CSI, the risks of treatment related long-term toxicity mandate a more cautious utilization of chemotherapy in cases with unequivocal evidence of GCT and when there is a clear benefit (13). Suboptimal management, either overor under-treatment, jeopardize the excellent outcomes of GCT patients and are equally harmful.

\section{Diagnosis of metastatic GCT}

Clinically, the majority of newly diagnosed GCT patients have clinical stage I (CSI) disease, which is defined by the absence of tumor outside the testis (14). Because of the lack of sensitive and specific biomarkers for relapse $(15,16)$, most of the CSI patients are managed with active surveillance (17). However, $15-20 \%$ of patients with CSI seminoma and $15-50 \%$ of patient with CSI non-seminoma will relapse and require active therapy (18). The diagnosis of metastatic GCT, either de novo or relapsed, is usually made by integration of clinical, imaging, and serologic tests.

\section{Imaging tests}

GCT have the advantage of demonstrating a very predictable pattern of metastases with the ipsilateral retroperitoneal lymph nodes being the first site of the metastatic spread in about $95 \%$ of the cases. Usually, nodules measuring $>1 \mathrm{~cm}$ are considered suspicious for metastatic disease (19). The sensitivity and specificity of the computer tomography (CT) scan is around $70 \%$ and is therefore purely based on morphologic criteria and limited by size criteria (20). Decreasing the cut off for significance increases the sensitivity but decreases the specificity, making this strategy not suitable for GCT patients where overtreatment is concerning because of the risk of longterm toxicity. Functional imaging tests (i.e., FDG-PET scan) have a very limited role in GCT; there are no data supporting their use in non-seminoma because of the known low FDG uptake of teratoma that results in a high rate of false negative results. In seminoma, FDG-PET has a relatively small utility in the post-chemotherapy setting for lesions $>3 \mathrm{~cm}$. As demonstrated by retrospective and prospective studies, in this setting FDG-PET scan has a high negative predictive value; however, the high false positive rate (around 70\%) limits the utilization of PET scan in this context for treatment decision, even if the results are positive $(21,22)$.

Although FDG-PET scan has been recently proposed to de-escalate metastatic seminoma patients treatment (23), the data are still controversial and insufficient to recommend the use of interim FDG-PET scan for treatment deescalation in seminoma patients.

\section{Serum tumor markers}

Although tumor markers (TM) alpha-fetoprotein (AFP), beta subunit of HCG (B-HCG) and LDH have a crucial role in GCT, their operating characteristics are also suboptimal in a number of clinical scenarios, contributing to a high degree of uncertainty (24). The detection of tumor markers in patients with GCT depends on the histology (higher sensitivity in non-seminoma than in seminoma) and tumor burden. Specifically, B-HCG is expressed by $<30 \%$ of seminoma patients while B-CG and AFP are expressed by $50-60 \%$ of non-seminoma patients (25).

Moreover, the half-life of AFP is around 7 days and

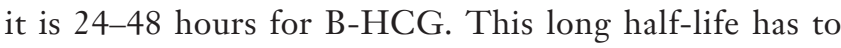
be considered when the patients are assessed for tumor response or residual disease on the base of the TM only since sometimes, especially when the initial levels were very high, they can be misinterpreted as falsely positive. Several non GCT related conditions are related to false positive TM elevation. Liver disease, liver toxicity, certain drugs, and some genetic conditions (hereditary persistence of AFP) lead to non GCT related increase of AFP. For B-HCG, false positive high levels have been described with use of marijuana, heterophile antibodies and high levels of LH hormones. LDH is the less specific of the GCT TM and can be increased for a variety of reasons including hemolysis or in case of G-CSF use (26). TM have diagnostic but also prognostic value and in fact they are part of the IGCCCG classification (27). Moreover, their levels are usually assessed during chemotherapy to monitor the response to the treatment. The TM decline rate identifies poor risk patients with better outcomes (28). The kinetics of TM after the first cycle of chemotherapy has been proposed to select the poor risk patients to treatment escalation in the GETUG 13 study (29). The updated results of the trial conducted in 263 patients, after a median follow-up of 5.6 years demonstrated that the patients with unfavorable TM decline treated with dose-dense chemotherapy had a better 5-year PFS [60\% vs. 47\%; HR: 0.65 (0.43-0.97); $\mathrm{P}=0.037$ while the 5 -year OS rate was not statistically significant [70.4\% vs. $60.8 \%$; HR: 0.69 (0.43-1.11); 
$\mathrm{P}=0.12]$ (30). This study confirmed the importance of $T M$ decline in poor risk patients although it did not provide clear definition of the best regimens for chemotherapy intensification.

\section{Objectives}

The objectives of this narrative review are: (I) to review the most relevant publications about micro-RNAs in GCTs; (II) to discuss the micro-RNAs potential clinical applications in patients with advanced GCTs. We present the following article in accordance with the Narrative Review reporting checklist (available at http://dx.doi. org/10.21037/tau-20-1246).

\section{Methods}

Literature review of the available publications was conducted searching "micro-RNA AND/IN germ cell tumors", "miR371a-3p in germ cell tumors" in PubMed.

\section{Narrative}

\section{New biomarkers in GCT: miRNAs}

miRNAs are small, non-coding RNAs of about 23 nucleotides involved in post-transcriptional gene regulation thus playing an essential role in many biological processes such as cell differentiation, apoptosis and tumor development $(31,32)$. Two clusters of miRNAs, miR-371a-3p (miR371) and miR-302/367, have been identified in embryonal stem cells where they control the expression of cell cycle regulating genes $(33,34)$. Voorhoeve and colleagues reported that in embryonic stem cells micro-RNAs play a pivotal role in the crossover between cell cycle control, pluripotency and chemo-sensitivity (35). These two clusters have also been described to be overexpressed in GCT tissue and cancer cell lines of both seminoma and non-seminoma while their expression is significantly lower in both benign testicular tissue and teratoma, demonstrating a high specificity for gem cell malignancy (36). miRNAs are usually secreted in the blood in multi-protein complexes that provide protection from RNAses degradation, increasing their stability in human body fluids (37). miR371 and miR302/367 clusters were identified in the serum of a pediatric patient with yolk sac tumor in 2011 by Dr. Murray's group for the first time (38). From that moment on, several studies have been published to demonstrate the detectability of those miRNAs in the blood of patients with GCT.
miRNAs in the pre-orchiectomy setting

The first studies were conducted as proof of principles in patients with testicular masses prior to and postorchiectomy. As reported by Belge first and confirmed by Gillis et al., serum miR-371/372/373/367 levels were significantly higher in the GCT samples than in the control samples (39). Moreover, these miRNA serum levels rapidly returned to baseline after orchiectomy in patients with CSI disease $(40,41)$. Those results were independently confirmed in a larger retrospective cohort of 250 patients with CSI GCT by van Agthoven et al. In this study, the test was performed prior to the orchiectomy and revealed a sensitivity of $90 \%$, a specificity of $86 \%$, a positive predictive value (PPV) of $94 \%$ and negative predictive value (NPV) of $79 \%$ as well as an Area Under the Curve (AUC) of the Receiver Operating Characteristic (ROC) of 0.95 for miR371 (42).

In their retrospective study, Spiekermann et al. demonstrated increased serum levels of miR371 in germ cell neoplasia in situ, stage I and metastatic GCT, compared with non-GCT control patients. Moreover, miR371 levels were significantly higher in the blood from the tumor ipsilateral testicular vein compared with the peripheral blood, confirming the high specificity of miR371 for GCT (43).

The largest retrospective study in patients with CSI pre and post orchiectomy was published by Dieckmann et al. (44). In the development cohort of this study, fifty patients with CSI GCT were evaluated for miR371 prior to and postorchiectomy. miR371 was compared to miR371 tested contextually to miR372, miR373 and miR367 and miR371 was confirmed to have the highest sensitivity and specificity with minimal additional benefit from combination with the other three miRNAs. The sensitivity and specificity of miR371 in the main study $(n=160)$ were $92 \%$ and $80 \%$, respectively. The study also demonstrated a direct correlation between GCT stage and levels of miR371 expression.

Those results were confirmed prospectively in a large study published by the same group in 2019 (45). Of the 616 patients evaluated, 522 had CSI GCT and miR371 was evaluated prior to the orchiectomy. In this setting the sensitivity of miR371 was $90.1 \%$, the specificity $94.0 \%$, the PPV $97.2 \%$, and the AUC of the ROC 0.966.

\section{Patients with metastatic disease}

Several studies have confirmed the high accuracy of circulating miR371 in detecting metastatic disease. In a small retrospective study in pediatric patients with GCT, 
Murray et al. demonstrated that miR371 and miR302/367 clusters are detectable in the cerebrospinal fluid as well as the serum of these patients with a sensitivity and specificity higher than the classic tumor markers (46).

In their retrospective study, Dieckmann et al. evaluated miR371 in 49 patients with metastatic GCT prior to, during and after chemotherapy (44). The miR371 levels decreased during chemotherapy but no data about detectable miR371 post-chemotherapy and presence of active germ cell malignancy (aGCM) were reported.

In 2019, two prospective studies were reported. Our group validated miR371 across the spectrum of GCTs (47). In this study 132 samples from 111 patients were assigned to three groups on the base of the risk of harboring aGCM. The low risk group (5-25\% risk) included patients with post-orchiectomy CSI seminoma and CSIA (negative lymphovascular invasion) non-seminoma with no suspicious signs of relapse on surveillance and patients with or without residual radiologic disease after chemotherapy and normal TM; the moderate risk group (25-50\% risk) included patients with post-orchiectomy CSIB (positive lymphovascular invasion) non-seminoma with no suspicious findings of relapse, patients with CSI seminoma and non-seminoma with clinical signs of suspicious relapse on surveillance, and patients with low positive TM after chemotherapy; the high risk group (90-100\% risk) included patients with gross clinical metastatic GCTs before starting chemotherapy, patients with testicular mass before orchiectomy, patients with CSIS and patients with obvious viable (high positive TM) residual disease after chemotherapy. Overall, with a median follow up of 15 months, specificity, and sensitivity of miR371 were $100 \%$ and $96 \%$, the positive predictive value and negative predictive value were $100 \%$, and $98 \%$, respectively. The AUC of miR371 was 0.96 , therefore significantly higher than the CT scan and classic tumor markers in all the clinical situations analyzed.

Another prospective study, analyzed miR371 in 118 patients with metastatic GCT, including 46 patients evaluated at the time of tumor relapse (45). The investigators confirmed that miR371 expression levels were associated with clinical stage, primary tumor size and response to the treatment. MiR371 median expression was higher in the relapsed patients than in controls and it was overexpressed in 38 of the 46 patients with GCT at the time of the clinically detectable relapse. No data about long-term follow-up were reported in this study.

Equivocal clinical scenarios and miRNAs utility
There are several situations where the suboptimal accuracy of the current diagnostic tests is highly problematic. These include stage IIA with either low positive or TM negative disease and patients presenting with post-chemotherapy residual disease.

\section{Stage IIA patients}

In absence of clearly elevated TM, the management of patients with $<2 \mathrm{~cm}$ retroperitoneal lymph nodes is challenging due to the high risk of falsely positive enlarged $\mathrm{LN}$ (48). In seminoma, enlarged $\mathrm{LN}$ can be related to inflammatory or granulomatous disease, known to have a certain association with this type of GCT (49). In nonseminoma, retrospective surgical series report that up to $40 \%$ of patients with $<2 \mathrm{~cm}$ retroperitoneal $\mathrm{LN}$ have no cancer and are potentially exposed to unnecessary treatments (50). Based on those data, a biomarker able to discriminate between absence and presence of aGCM is critical.

The operating characteristics of miR371 in patients with CSI and stage IIA have been evaluate by Lafin et al. in 24 patients with seminoma and non-seminoma treated with primary RPLND (51). The serum levels of miR371 were correlated with the presence or absence of active GCT in the pathology specimens. Of the 24 patients analyzed, 11 $(45.8 \%)$ presented aGCM, $10(41.7 \%)$ benign tissue and 3 (12.5\%) teratoma. miR371 demonstrated high sensitivity (100\%) and specificity (92\%) to detect aGCM, with an AUC of the ROC of 0.96 in this very equivocal clinical scenario.

The results in moderate risk patients evaluated in our study also confirmed the high accuracy and clinical validity of miR371 in patients with equivocal GCT evidence (47). In the 46 patients with moderate risk analyzed, miR371 showed a sensitivity, specificity, NPV and PPV of $92 \%$, $100 \%, 97 \%$ and $100 \%$ (none of the patients had a false positive result), respectively. The operating characteristics of miR371 in identifying aGCM outperformed CT imaging and TM in this diagnostically very problematic group of patients with an AUC of the ROC of 0.89 .

\section{Post-chemotherapy residual disease}

The management of post-chemotherapy residual disease depends on histology and size of disease. In seminoma, the risk of viable seminoma is low if the residual disease is $\leq 3 \mathrm{~cm}$. In lesions $>3 \mathrm{~cm}$ the risk of viable residual seminoma is about $20-30 \%$ (52). FDG-PET has high 
negative predictive value but the positive predictive value is only around $30 \%(21,22,53)$. In addition, TM are commonly negative in seminoma and do not contribute to characterize the presence of aGCM. In nonseminoma, surgery is recommended for every patient with residual lesions $>1 \mathrm{~cm}(54,55)$. However, the analysis of retrospective large surgical series in this patients population have revealed that only $10 \%$ of patients have residual aGCM, while $40-50 \%$ of the patients have teratoma and $40-50 \%$ have necrosis/fibrosis only (56). Overtreatment is therefore implied in the significant group of patients with non-seminoma without postchemotherapy residual teratoma or viable GCT who were cured with chemotherapy alone but who cannot currently be differentiated from patients with teratoma or aGCM on the base of the imaging studies.

Several clinical nomograms have been proposed to identify the patients with necrosis/scar tissue only residual disease post-chemotherapy (57-59). However, the accuracy of those strategies is highly suboptimal and cannot and should not be routinely used for management decisions.

In this setting, a study reported by Leao et al. has demonstrated that miR371 is able to detect the presence of aGCM also in patients with post-chemotherapy residual disease (60). The study was conducted in 82 GCT patients with residual disease after chemotherapy and prior to RPLND and the serum miRNAs expression was correlated with the pathology results of the RPLND specimens. With the limitation of having only 12 patients harboring aGCM after chemotherapy, miR371 was able to predict the presence of aGCM with a specificity of $50 \%$ and sensitivity of $100 \%$. The AUC of miR371 alone was 0.874 , comparable to the combined miRNAs 371, 372 and 373 (AUC 0.885). As previously reported, miR371 was undetectable in patients with residual teratoma or fibrosis.

Serum miR371/373/367 were also analyzed by Rosaspost-chemotherapy in a cohort of 109 patients (61). The median level of these miRNAs was higher in patients who developed a relapse $(n=34)$ than in those who had complete durable remission $(n=60)$. Moreover, serum miR367 was higher in cisplatin refractory patients $(\mathrm{n}=15)$ compared to the patients who achieved a complete response.

As demonstrated for primary testicular masses and metastatic disease $(44,45,62)$, it is very likely that the sensitivity of miR371 in detecting aGCM also in the postchemotherapy setting relies on the residual aGCM tumor burden after chemotherapy.

\section{Other equivocal scenarios}

Considering the remarkably high specificity of miR371 in identifying aGCM, it could be clinically valuable to detect GCT in patients with metastatic primary unknown tumors. Especially in absence of elevated serum tumor markers and/ or obvious testicular masses, the diagnosis of GCT maybe challenging and requiring invasive procedure to collect tissue for pathology diagnosis. miR371 could be used in this very equivocal cases to identify aGCM and therefore to offer curative treatments.

\section{Limitations of miR371}

In order to promote the scalability and implementation of miR371, standard methodology should be used for miRNAs extraction and expression analysis. Normalization of miR371 using internal housekeeping miRNAs is recommended to reduce the variability of results related to technical issues. Moreover, hemolysis should be always considered as it could affect the miRNAs expression results $(46,63)$. Although miR371 is potentially useful to predict the presence of aGCM, in the post-chemotherapy setting its utility is limited by the negative expression in teratoma. As a consequence, while patients with positive miR371 have high risk of having aGCM in case of negative serum/ plasma miR371 the presence of teratoma cannot be rule out. Therefore, several efforts have been made to identify biomarkers specific for teratoma to be integrated with miR371 to characterize the pathology composition of residual post-chemotherapy masses.

\section{Potential teratoma biomarkers: miR375-3p}

miR375-3p (miR375) is a miRNA overexpressed in teratoma and yolk sac tumor tissue (64). Unlike miR371, this miRNA is not specific only for teratoma or GCT but low levels of miR375 are detectable in healthy volunteer (no cancer patients) (65) and it is overexpressed also in other solid malignancies (66). The detectability of miR375 in the blood of patients with GCT has been reported in 4 studies but with controversial results.

Belge et al. reported about serum miR375 in 21 patients with teratoma, 12 patients with other GCT, and 12 control samples (67). The levels of miR375 were not different between teratoma and other GCT patients or controls. The AUC of the ROC for miR375 in identifying teratoma was 0.524 .

The serum miR375 was retrospectively analyzed by Lobo et al. in a cohort 36 patients undergoing chemotherapy 
followed by RPLND at different time points: prechemotherapy, post-chemotherapy, pre-RPLND and postRPLND (68). 15/36 patients presented teratoma and no significant differences were found between them and the patients with benign or residual aGCM prior to or postchemotherapy or post-RPLND.

Kenigsberg et al. recently reported about the serum expression of miRNA-375 and miR375-5p prospectively evaluated in 40 GCT patients (19 teratoma, 18 benign pathology, 2 viable GCT, 1 embryonal rhabdomyosarcoma) who underwent post-chemotherapy RPLND (69). miR$375-5 p$ was undetectable in all samples examined. The AUC of the ROC for miR375 was 0.503 . Of the 19 patients with teratoma, 16 had elevated miRNA-375 with a sensitivity of $84.2 \%$; the specificity was $35.3 \%$. PPV and NPV were $59.3 \%$ and $66.7 \%$, respectively.

Our group proposed an integrated analysis of miR371 and miR375 to predict the risk of aGCM and teratoma in patients with post-chemotherapy residual disease. In our study, plasma miR375 and miR371 were analyzed in 41 patients with confirmed pure teratoma and compared with patients with either no tumor or pure classic seminoma $(n=59)$ (70). Overall, sensitivity and specificity of miR375 in identifying teratoma were $78 \%$ and $80 \%$, respectively; the AUC was 0.7. However, the integrated analysis of both miR371 and miR375 increased the discriminative power to distinguish benign to teratoma and aGCM tissue in patients with residual disease post-chemotherapy (70). Although small and preliminary, our data suggest that there is a potential in developing an integrated clinical-molecular model to predict teratoma, aGCM and benign tissue in patients with post-chemotherapy residual disease that may increase the accuracy of the current diagnostic tools.

Methodologic differences among the above mentioned studies may contribute to explain the difference in the results. The collecting tubes were different. While Nappi et al. used cell-free DNA BCT (Streck tubes) and plasma for their analysis, in the other three studies the blood was collected in serum separator tubes and miR375 was analyzed in serum. Although no data about accuracy detection of miR375 in cell-free DNA BCT tubes vs. serum separator tubes are available, some evidence suggest that preservation solutions for cell-free DNA designed to prevent cell lysis and apoptosis also minimize release of cellular miRNAs, reducing the unwanted background after blood collection and improving the detection of the targeted miRNAs of interest (71).

Moreover, while Nappi et al. demonstrated a clear correlation between the size of the teratoma component and the plasma expression of miR375, no data regarding the correlation between the size of the teratoma in the post-chemotherapy residual disease and miR375 level of expression were provided in the other studies. This is relevant and may explain the differences of the results observed in the above mentioned studies. Furthermore, the number of the teratoma patients included in each study was different: in the Belge publication only 5 patients with teratoma had CS II-III while the majority of patients had CSI disease. In Lobo's paper, it is unclear from the manuscript whether or not the teratoma patients had only pure teratoma or included also patients with mixed histology.

\section{Status of miRNAs validation in GCT}

Although several independent groups have demonstrated the high accuracy of miR371 in detecting aGCM, there is still the need to further validate this miRNA in larger prospective trials before introducing its utilization in the clinical practice. While its clinical validity has been demonstrated in patients with obvious disease (preorchiectomy and in patients with unequivocal metastatic disease) the operating characteristics of this miRNA in patients with more equivocal disease or microscopic disease are still unknown.

In order to validate the clinical utility of miR371 in the diagnosis and follow up of GCT patients and to define the cut off of sensitivity to detect GCT, two prospective clinical trials have been designed and are currently enrolling patients.

The Children's Oncology Group AGCT 1523 trial (Active Surveillance, Bleomycin, Carboplatin, Etoposide, or Cisplatin in Treating Pediatric and Adult Patients With Germ Cell Tumors) is a multi-arms study designed in the pediatric population with an optional biomarker arm enrolling also adult GCT patients to evaluate $\mathrm{miR} 371 / \mathrm{miR} 372 / \mathrm{miR} 373$ and miR367 clusters during the surveillance of CSI patients (72).

In adult GCT patients, S1823, a SWOG international cohort clinical trial, has been activated in June 2020 and is currently recruiting GCT patients (73). This study primary objective is to correlate miR371 to the presence of relapse in patients with CSI and CSIIA on surveillance; the primary endpoint is to establish the PPV of miR371 in detecting GCT relapse. Since the study plans serial blood tests, it will be possible to establish if and when exactly miR371 
becomes positive during surveillance and to correlate the miR371 status with the clinical presentation of the disease at the time of tumor relapse.

\section{Future prospective}

In addition to S1823, another clinical trial has been planned to validate the use of miR371 expression in patients with GCT with $<3 \mathrm{~cm}$ retroperitoneal $\mathrm{LN}$ to select patients for either surgery or observation. Moreover, there is a lot of enthusiasm in the GCT community for joint efforts to build up integrated clinical and biomarkers models for the identification of teratoma and benign residual disease in patients with non-seminoma presenting with postchemotherapy residual disease. In this setting, radiomics has showed promising results and need to be further explored $(74,75)$.

\section{Conclusions}

In conclusion, miR371 has the potential to increase the accuracy of detecting GCT and therefore to personalize the treatment of patients with this disease. There are immediate and clinically meaningful consequences if miR371 utility is clinically validated. This biomarker can minimize the rate of suboptimal treatments reducing long-term toxicity related to unnecessary treatments and therefore improving the quality of life and the life expectancy of the young GCT patients.

\section{Acknowledgments}

Funding: None.

\section{Footnote}

Provenance and Peer Review: This article was commissioned by the Guest Editor (Tilman Todenhöfer) for the series "Management of Advanced Genitourinary Malignancies" published in Translational Andrology and Urology. The article has undergone external peer review.

Reporting Checklist: The authors have completed the Narrative Review reporting checklist. Available at http:// dx.doi.org/10.21037/tau-20-1246

Peer Review File: Available at http://dx.doi.org/10.21037/ tau-20-1246
Conflicts of Interest: All authors have completed the ICMJE uniform disclosure form (available at http://dx.doi. org/10.21037/tau-20-1246). The series "Management of Advanced Genitourinary Malignancies" was commissioned by the editorial office without any funding or sponsorship. LN reports other from Bayer, other from Pfizer, other from Ipsen, other from Astellas, outside the submitted work. The authors have no other conflicts of interest to declare.

Ethical Statement: The authors are accountable for all aspects of the work in ensuring that questions related to the accuracy or integrity of any part of the work are appropriately investigated and resolved.

Open Access Statement: This is an Open Access article distributed in accordance with the Creative Commons Attribution-NonCommercial-NoDerivs 4.0 International License (CC BY-NC-ND 4.0), which permits the noncommercial replication and distribution of the article with the strict proviso that no changes or edits are made and the original work is properly cited (including links to both the formal publication through the relevant DOI and the license). See: https://creativecommons.org/licenses/by-nc-nd/4.0/.

\section{References}

1. Goldberg K. ASCO 50th anniversary poll names the top 5 advances from the past 50 years. 2014. Available online: https://www.asco.org/about-asco/press-center/ news-releases/asco-50th-anniversary-poll-names-top-5advances-past-50-years

2. Einhorn LH. Treatment of testicular cancer: a new and improved model. J Clin Oncol 1990;8:1777-81.

3. Einhorn LH. Testicular cancer as a model for a curable neoplasm: The Richard and Hinda Rosenthal Foundation Award Lecture. Cancer Res 1981;41:3275-80.

4. Kollmannsberger C, Tyldesley S, Moore C, et al. Evolution in management of testicular seminoma: population-based outcomes with selective utilization of active therapies. Ann Oncol 2011;22:808-14.

5. Tandstad T, Kollmannsberger CK, Roth BJ, et al. Practice Makes Perfect: The Rest of the Story in Testicular Cancer as a Model Curable Neoplasm. J Clin Oncol 2017;35:3525-8.

6. Kerns SL, Fung C, Monahan PO, et al. Cumulative Burden of Morbidity Among Testicular Cancer Survivors After Standard Cisplatin-Based Chemotherapy: A MultiInstitutional Study. J Clin Oncol 2018;36:1505-12. 
7. Agrawal V, Dinh PC, Jr., Fung C, et al. Adverse Health Outcomes Among US Testicular Cancer Survivors After Cisplatin-Based Chemotherapy vs. Surgical Management. JNCI Cancer Spectr 2020;4:pkz079.

8. Travis LB, Ng AK, Allan JM, et al. Second malignant neoplasms and cardiovascular disease following radiotherapy. J Natl Cancer Inst 2012;104:357-70.

9. Huddart RA, Norman A, Shahidi M, et al. Cardiovascular disease as a long-term complication of treatment for testicular cancer. J Clin Oncol 2003;21:1513-23.

10. Fung C, Sesso HD, Williams AM, et al. MultiInstitutional Assessment of Adverse Health Outcomes Among North American Testicular Cancer Survivors After Modern Cisplatin-Based Chemotherapy. J Clin Oncol 2017;35:1211-22.

11. Fossa SD, Gilbert E, Dores GM, et al. Noncancer causes of death in survivors of testicular cancer. J Natl Cancer Inst 2007;99:533-44.

12. Alanee SR, Feldman DR, Russo P, et al. Long-term mortality in patients with germ cell tumors: effect of primary cancer site on cause of death. Urol Oncol 2014;32:26.e9-15.

13. Nichols C, Nappi L, Kollmannsberger C, et al. Zero, Some Chemotherapy Game in Early-Stage Germ Cell Tumors. J Clin Oncol 2020;38:2214-5.

14. Powles TB, Bhardwa J, Shamash J, et al. The changing presentation of germ cell tumours of the testis between 1983 and 2002. BJU Int 2005;95:1197-200.

15. Chung P, Daugaard G, Tyldesley S, et al. Evaluation of a prognostic model for risk of relapse in stage I seminoma surveillance. Cancer Med 2015;4:155-60.

16. Albers P, Siener R, Kliesch S, et al. Risk factors for relapse in clinical stage I nonseminomatous testicular germ cell tumors: results of the German Testicular Cancer Study Group Trial. J Clin Oncol 2003;21:1505-12.

17. Nichols CR, Roth B, Albers P, et al. Active surveillance is the preferred approach to clinical stage I testicular cancer. J Clin Oncol 2013;31:3490-3.

18. Kollmannsberger C, Tandstad T, Bedard PL, et al. Patterns of relapse in patients with clinical stage I testicular cancer managed with active surveillance. J Clin Oncol 2015;33:51-7.

19. Brunereau L, Bruyere F, Linassier C, et al. The role of imaging in staging and monitoring testicular cancer. Diagn Interv Imaging 2012;93:310-8.

20. Dalal PU, Sohaib SA, Huddart R. Imaging of testicular germ cell tumours. Cancer Imaging 2006;6:124-34.

21. De Santis M, Becherer A, Bokemeyer C, et al. 2-18fluoro-
deoxy-D-glucose positron emission tomography is a reliable predictor for viable tumor in postchemotherapy seminoma: an update of the prospective multicentric SEMPET trial. J Clin Oncol 2004;22:1034-9.

22. Cathomas R, Klingbiel D, Bernard B, et al. Questioning the Value of Fluorodeoxyglucose Positron Emission Tomography for Residual Lesions After Chemotherapy for Metastatic Seminoma: Results of an International Global Germ Cell Cancer Group Registry. J Clin Oncol 2018. [Epub ahead of print].

23. Loriot $\mathrm{Y}$, Texier M, Culine S, et al. The SEMITEP trial: De-escalating chemotherapy in low-volume metastatic seminoma based on early FDG-PET. J Clin Oncol 2020;38:387.

24. Gilligan TD, Hayes DF, Seidenfeld J, et al. ASCO Clinical Practice Guideline on Uses of Serum Tumor Markers in Adult Males With Germ Cell Tumors. J Oncol Pract 2010;6:199-202.

25. Dieckmann KP, Simonsen-Richter H, Kulejewski M, et al. Serum Tumour Markers in Testicular Germ Cell Tumours: Frequencies of Elevated Levels and Extents of Marker Elevation Are Significantly Associated with Clinical Parameters and with Response to Treatment. Biomed Res Int 2019;2019:5030349.

26. Milose JC, Filson CP, Weizer AZ, et al. Role of biochemical markers in testicular cancer: diagnosis, staging, and surveillance. Open Access J Urol 2011;4:1-8.

27. International Germ Cell Consensus Classification: a prognostic factor-based staging system for metastatic germ cell cancers. International Germ Cell Cancer Collaborative Group. J Clin Oncol 1997;15:594-603.

28. Fizazi K, Culine S, Kramar A, et al. Early predicted time to normalization of tumor markers predicts outcome in poor-prognosis nonseminomatous germ cell tumors. J Clin Oncol 2004;22:3868-76.

29. Fizazi K, Pagliaro L, Laplanche A, et al. Personalised chemotherapy based on tumour marker decline in poor prognosis germ-cell tumours (GETUG 13): a phase 3, multicentre, randomised trial. Lancet Oncol 2014;15:1442-50.

30. Fizazi K, Flechon A, Le Teuff G, et al. Mature results of the GETUG 13 phase III trial in poor-prognosis germcell tumors (GCT). J Clin Oncol 2016;34:4504.

31. Peng Y, Croce CM. The role of MicroRNAs in human cancer. Signal Transduct Target Ther 2016;1:15004.

32. Zamore PD, Haley B. Ribo-gnome: the big world of small RNAs. Science 2005;309:1519-24.

33. Eini R, Dorssers LC, Looijenga LH. Role of stem cell 
proteins and microRNAs in embryogenesis and germ cell cancer. Int J Dev Biol 2013;57:319-32.

34. Jesus ABD, Lucena-Aguilar G, Menendez P. The miR302-367 cluster as a potential stemness regulator in ESCs. Cell Cycle 2009;8:394-8.

35. Voorhoeve PM, le Sage C, Schrier M, et al. A genetic screen implicates miRNA-372 and miRNA-373 as oncogenes in testicular germ cell tumors. Cell 2006;124:1169-81.

36. Palmer RD, Murray MJ, Saini HK, et al. Malignant germ cell tumors display common microRNA profiles resulting in global changes in expression of messenger RNA targets. Cancer Res 2010;70:2911-23.

37. Li L, Zhu D, Huang L, et al. Argonaute 2 complexes selectively protect the circulating microRNAs in cellsecreted microvesicles. PLoS One 2012;7:e46957.

38. Murray MJ, Halsall DJ, Hook CE, et al. Identification of microRNAs From the miR-371 373 and miR-302 clusters as potential serum biomarkers of malignant germ cell tumors. Am J Clin Pathol 2011;135:119-25.

39. Belge G, Dieckmann KP, Spiekermann M, et al. Serum levels of microRNAs miR-371-3: a novel class of serum biomarkers for testicular germ cell tumors? Eur Urol 2012;61:1068-9.

40. Gillis AJ, Rijlaarsdam MA, Eini R, et al. Targeted serum miRNA (TSmiR) test for diagnosis and follow-up of (testicular) germ cell cancer patients: a proof of principle. Mol Oncol 2013;7:1083-92.

41. Radtke A, Hennig F, Ikogho R, et al. The Novel Biomarker of Germ Cell Tumours, Micro-RNA-371a-3p, Has a Very Rapid Decay in Patients with Clinical Stage 1. Urol Int 2018;100:470-5.

42. van Agthoven T, Looijenga LHJ. Accurate primary germ cell cancer diagnosis using serum based microRNA detection (ampTSmiR test). Oncotarget 2017;8:58037-49.

43. Spiekermann M, Belge G, Winter N, et al. MicroRNA miR-371a-3p in serum of patients with germ cell tumours: evaluations for establishing a serum biomarker. Andrology 2015;3:78-84.

44. Dieckmann KP, Radtke A, Spiekermann M, et al. Serum Levels of MicroRNA miR-371a-3p: A Sensitive and Specific New Biomarker for Germ Cell Tumours. Eur Urol 2017;71:213-20.

45. Dieckmann KP, Radtke A, Geczi L, et al. Serum Levels of MicroRNA-371a-3p (M371 Test) as a New Biomarker of Testicular Germ Cell Tumors: Results of a Prospective Multicentric Study. J Clin Oncol 2019;37:1412-23.

46. Murray MJ, Bell E, Raby KL, et al. A pipeline to quantify serum and cerebrospinal fluid microRNAs for diagnosis and detection of relapse in paediatric malignant germ-cell tumours. Br J Cancer 2016;114:151-62.

47. Nappi L, Thi M, Lum A, et al. Developing a Highly Specific Biomarker for Germ Cell Malignancies: Plasma miR371 Expression Across the Germ Cell Malignancy Spectrum. J Clin Oncol 2019;37:3090-8.

48. Kollmannsberger CK, Nappi L, Nichols C. Management of Stage II Germ Cell Tumors: Be Sure, Be Patient, Be Safe. J Clin Oncol 2019;37:1856-62.

49. Tjan-Heijnen VC, Vlasveld LT, Pernet FP, et al. Coincidence of seminoma and sarcoidosis: a myth or fact? Ann Oncol 1998;9:321-5.

50. Stephenson AJ, Bosl GJ, Motzer RJ, et al. Nonrandomized comparison of primary chemotherapy and retroperitoneal lymph node dissection for clinical stage IIA and IIB nonseminomatous germ cell testicular cancer. J Clin Oncol 2007;25:5597-602.

51. Lafin JT, Singla N, Woldu SL, et al. Serum MicroRNA371a-3p Levels Predict Viable Germ Cell Tumor in Chemotherapy-naive Patients Undergoing Retroperitoneal Lymph Node Dissection. Eur Urol 2020;77:290-2.

52. Ravi R, Ong J, Oliver RT, et al. The management of residual masses after chemotherapy in metastatic seminoma. BJU Int 1999;83:649-53.

53. Bachner M, Loriot Y, Gross-Goupil M, et al. 2-(1)(8) fluoro-deoxy-D-glucose positron emission tomography (FDG-PET) for postchemotherapy seminoma residual lesions: a retrospective validation of the SEMPET trial. Ann Oncol 2012;23:59-64.

54. Daneshmand S, Stephenson AJ, Sheinfeld J, et al. The management of subcentimeter residual mass in NSGCT: pcRPLND vs. observation. Urol Oncol 2011;29:842-7.

55. Ravi P, Gray KP, O'Donnell EK, et al. A meta-analysis of patient outcomes with subcentimeter disease after chemotherapy for metastatic non-seminomatous germ cell tumor. Ann Oncol 2014;25:331-8.

56. Heidenreich A, Pfister D, Witthuhn R, et al. Postchemotherapy retroperitoneal lymph node dissection in advanced testicular cancer: radical or modified template resection. Eur Urol 2009;55:217-24.

57. Leao R, Nayan M, Punjani N, et al. A New Model to Predict Benign Histology in Residual Retroperitoneal Masses After Chemotherapy in Nonseminoma. Eur Urol Focus 2018;4:995-1001.

58. Vergouwe Y, Steyerberg EW, Foster RS, et al. Predicting retroperitoneal histology in postchemotherapy testicular germ cell cancer: a model update and multicentre validation with more than 1000 patients. Eur Urol 
2007;51:424-32.

59. Paffenholz P, Nestler T, Hoier S, et al. External validation of 2 models to predict necrosis/fibrosis in postchemotherapy residual retroperitoneal masses of patients with advanced testicular cancer. Urol Oncol 2019;37:809.e9-e18.

60. Leao R, van Agthoven T, Figueiredo A, et al. Serum miRNA Predicts Viable Disease after Chemotherapy in Patients with Testicular Nonseminoma Germ Cell Tumor. J Urol 2018;200:126-35.

61. Rosas Plaza X, van Agthoven T, Meijer C, et al. miR-371a$3 p$, miR-373-3p and miR-367-3p as Serum Biomarkers in Metastatic Testicular Germ Cell Cancers Before, During and After Chemotherapy. Cells 2019;8:1221.

62. Syring I, Bartels J, Holdenrieder S, et al. Circulating serum miRNA (miR-367-3p, miR-371a-3p, miR-372-3p and $\mathrm{miR}-373-3 \mathrm{p}$ ) as biomarkers in patients with testicular germ cell cancer. J Urol 2015;193:331-7.

63. Myklebust MP, Rosenlund B, Gjengsto P, et al. Quantitative PCR Measurement of miR-371a-3p and miR-372-p Is Influenced by Hemolysis. Front Genet 2019;10:463.

64. Shen H, Shih J, Hollern DP, et al. Integrated Molecular Characterization of Testicular Germ Cell Tumors. Cell Rep 2018;23:3392-406.

65. Zhang H, Yang H, Zhang C, et al. Investigation of microRNA expression in human serum during the aging process. J Gerontol A Biol Sci Med Sci 2015;70:102-9.

66. Yan JW, Lin JS, He XX. The emerging role of miR-375 in cancer. Int J Cancer 2014;135:1011-8.

67. Belge G, Grobelny F, Matthies C, et al. Serum Level of microRNA-375-3p Is Not a Reliable Biomarker of

Cite this article as: Nappi L, Nichols C, Kollmannsberger C. Narrative review of developing new biomarkers for decision making in advanced testis cancer. Transl Androl Urol 2021;10(10):4075-4084. doi: 10.21037/tau-20-1246
Teratoma. In Vivo 2020;34:163-8.

68. Lobo J, Gillis AJM, van den Berg A, et al. Identification and Validation Model for Informative Liquid BiopsyBased microRNA Biomarkers: Insights from Germ Cell Tumor In Vitro, In Vivo and Patient-Derived Data. Cells 2019;8:1637.

69. Kenigsberg A, Lafin J, Meng XS, et al. Predictive Capacity of Mirna-375 in Identifying Teratoma in PostChemotherapy Retroperitoneal Lymph Node Dissection (Pc-Rplnd). J Urology 2020;203:E385.

70. Nappi L, Thi M, Adra N, et al. Integrated Expression of Circulating miR375 and miR371 to Identify Teratoma and Active Germ Cell Malignancy Components in Malignant Germ Cell Tumors. Eur Urol 2021;79:16-9.

71. Grolz D, Hauch S, Schlumpberger M, et al. Liquid Biopsy Preservation Solutions for Standardized Pre-Analytical Workflows-Venous Whole Blood and Plasma. Current Pathobiology Reports 2018;6:275-86.

72. ClinicalTrials.gov. Identifier: NCT03067181. Available online: https://clinicaltrials.gov/ct2/show/NCT03067181

73. ClinicalTrials.gov. Identifier: NCT04435756. Available online: https://clinicaltrials.gov/ct2/show/NCT04435756

74. Lewin J, Dufort P, Halankar J, et al. Applying Radiomics to Predict Pathology of Postchemotherapy Retroperitoneal Nodal Masses in Germ Cell Tumors. JCO Clin Cancer Inform 2018;2:1-12.

75. Baessler B, Nestler T, Pinto Dos Santos D, et al. Radiomics allows for detection of benign and malignant histopathology in patients with metastatic testicular germ cell tumors prior to post-chemotherapy retroperitoneal lymph node dissection. Eur Radiol 2020;30:2334-45. 